\title{
Boundedly solvable degenerate differential operators for first order
}

\author{
Pembe İpek $^{\mathrm{a}}$ and Zameddin İ. İsmailov ${ }^{\mathrm{b}^{*}}$ \\ anstitute of Natural Sciences, Karadeniz Technical University, Turkey \\ ${ }^{\mathrm{b}}$ Department of Mathematics, Karadeniz, Technical University, Turkey \\ *Corresponding author E-mail: zameddin.ismailov@gmail.com
}

\section{Article Info}

Keywords: Boundedly solvable operator, Degenerate differential-operator expression, Spectrum

2010 AMS: 47A20,47B38

Received: 31 January 2018

Accepted: 23 February 2018

Available online: 11 March 2018

\begin{abstract}
Using the methods of operator theory all boundedly solvable extensions of the minimal operator generated by degenerated type differential-operator expression in the weighted Hilbert space of vector-functions in finite interval in terms of boundary conditions are described. Later on, the structure of spectrum of these type extensions will be investigated.
\end{abstract}

\section{Introduction}

The general information on the degenerate differential equations in Banach spaces can be found in book of A. Favini and A. Yagi [1]. The fundamental interest to such equations are motivated by applications in different fields of life sciences.

Recall that an operator

$$
A: D(A) \subset H \rightarrow H
$$

in a Hilbert space $H$ is called boundedly solvable, if $A$ is one-to-one

$$
A D(A)=H \text { and } A^{-1} \in L(H) .
$$

In this work using the methods of operator theory, all boundedly solvable extensions of minimal operator generated by linear degenerate type differential-operator expression in the weighted Hilbert space of vector-functions in finite interval in terms of boundary conditions have been defined (see Sec.2). In Section 3 the geometry of spectrum set of these type extensions has been investigated.

Let $H$ be a separable Hilbert space and $\alpha:(0,1) \rightarrow(0, \infty), \alpha \in C(0,1)$ and $\int_{0}^{1} \frac{d t}{\alpha(t)}<\infty$. In the weighted Hilbert space $L_{\alpha}^{2}(H,(0,1))$ of $H-$ valued vector-functions defined at the interval $(0,1)$ consider the following degenerate type differential expression with operator coefficient for first order in a form

$$
l(u)=(\alpha u)^{\prime}(t)+A(t) u(t)
$$

where:

(1) operator-function $A(\cdot):(0,1) \rightarrow L(H)$ is continuous on the uniform operator topology;

(2) $\frac{\|A(t)\|}{\alpha(t)} \in L^{1}(0,1)$.

By the standard way the minimal $L_{0}$ and maximal $L$ operators corresponding differential expression $l(\cdot)$ in $L_{\alpha}^{2}(H,(0,1))$ can be defined [3]. In this case $\operatorname{Ker} L_{0}=\{0\}$ and $\overline{\operatorname{Im}\left(L_{0}\right)} \neq L_{\alpha}^{2}(H,(0,1))$ (see Sec.3).

In this work, firstly all boundedly solvable extensions of the minimal operator generated by first order linear degenerate type differentialoperator expression in the weighted Hilbert space of vector-functions in $(0,1)$ in terms of boundary conditions are described. Later on, the structure of spectrum of these type extensions will be investigated. 


\section{Description of boundedly solvable extensions}

In this section using the Vishik's methods all boundedly solvable extensions of the minimal operator generated by linear degenerate type differential-operator expression $l(\cdot)$ in weighted Hilbert space $L_{\alpha}^{2}(H,(0,1))$ are represented.

Before of all note that using the knowing standard way the minimal $M_{0}$ and the maximal $M$ operators generated by differential expression

$$
m(v)=(\alpha v)^{\prime}(t)
$$

in Hilbert space $L_{\alpha}^{2}(H,(0,1))$ can be defined [3].

Later on, by $U(t, s), t, s \in[0,1)$ will be defined the family of evolution operators corresponding to the homogeneous differential-operator equation

$$
\alpha(t) \frac{\partial}{\partial t} U(t, s) f+A(t) U(t, s) f=0, t, s \in(0,1)
$$

with boundary condition

$$
U(s, s) f=f, f \in H .
$$

The operator $U(t, s), t, s \in(0,1)$ is linear continuous and boundedly solvable in $H$. And also for any $t, s \in(0,1)$ there is the following equation:

$$
U^{-1}(t, s)=U(s, t)
$$

(for detail analysis see [2]).

If introduce the following operator

$$
\begin{aligned}
& U z(t)=U(t, 0) z(t), \\
& U: L_{\alpha}^{2}(H,(0,1)) \rightarrow L_{\alpha}^{2}(H,(0,1)),
\end{aligned}
$$

then it is easily to check that

$$
\begin{aligned}
l(U z) & =(\alpha U z)^{\prime}(t)+A(t) U z(t) \\
& =U(\alpha z)^{\prime}(t)+U_{t}^{\prime}(\alpha z)(t)+A(t) U z(t) \\
& =U(\alpha z)^{\prime}(t)+\left[\alpha(t) U_{t}^{\prime} z(t)+A(t) U z(t)\right] \\
& =U(\alpha z)^{\prime}(t) \\
& =U m(z) .
\end{aligned}
$$

Therefore it can be obtained

$$
U^{-1} l(U z)=m(z)
$$

Hence it is clear that if $\widetilde{L}$ is some extension of the minimal operator $L_{0}$, that is, $L_{0} \subset \widetilde{L} \subset L$, then $U^{-1} L_{0} U=M_{0}, M_{0} \subset U^{-1} \widetilde{L} U=\widetilde{M} \subset$ $M, U^{-1} L U=M$.

Now we prove the following assertion.

Theorem 2.1. $\operatorname{Ker} L_{0}=\{0\}$ and $\overline{\operatorname{Im}\left(L_{0}\right)} \neq L_{\alpha}^{2}(H,(0,1))$.

Proof. Consider the following boundary value problem in $L_{\alpha}^{2}(H,(0,1))$

$$
\begin{aligned}
& (\alpha u)^{\prime}(t)+A(t) u(t)=0, t \in(0,1), \\
& (\alpha u)(0)=(\alpha u)(1)=0 .
\end{aligned}
$$

Then the general solution of above differential equation is in form

$$
(\alpha u)(t)=\exp \left(-\int_{0}^{t} \frac{A(s)}{\alpha(s)} d s\right) f_{0}, f_{0} \in H .
$$

From (2.7) and boundary condition (2.6) we have following equation

$$
u(t)=0, t \in(0,1) .
$$

Consequently, following equality $\operatorname{Ker}\left(L_{0}\right)=\{0\}$ hold.

On the other hand it is clear that the general solution of following differential equation in $L_{\alpha}^{2}(H,(0,1))$

$$
-(\alpha v)^{\prime}(t)+A^{*}(t) v(t)=0
$$

in form

$$
v(t)=\frac{1}{\alpha(t)} \exp \left(\int_{0}^{t} \frac{A^{*}(s)}{\alpha(s)} d s\right) g, g \in H
$$


This means that

$$
\operatorname{dim} \operatorname{Ker} L_{0}^{*}=\infty .
$$

So the following inequality is realized

$$
\overline{\operatorname{Im}\left(L_{0}\right)} \neq L_{\alpha}^{2}(H,(0,1)) .
$$

Theorem 2.2. Each solvable extension $\widetilde{L}$ of the minimal operator $L_{0}$ in $L_{\alpha}^{2}(H,(0,1))$ is generated by the differential-operator expression $l(\cdot)$ with boundary condition

$$
(B+E)\left(\alpha U^{-1} u\right)(0)=B\left(\alpha U^{-1} u\right)(1),
$$

where $B \in L(H), E$ is a identity operator in $H$. The operator $B$ is determined uniquely by the extension $\widetilde{L}$, i.e $\widetilde{L}=L_{B}$.

On the contrary, the restriction of the maximal operator $L$ to the manifold of vector-functions satisfy the above boundary condition for some bounded operator $B \in L(H)$ is a boundedly solvable extension of the minimal operator $L_{0}$ in $L_{\alpha}^{2}(H,(0,1))$.

Proof. Firstly, all boundedly solvable extensions $\widetilde{M}$ of the minimal operator $L_{0}$ in $L_{\alpha}^{2}(H,(0,1))$ in terms of boundary conditions will be described.

Consider the following so-called Cauchy extension $M_{c}$,

$$
\begin{aligned}
& M_{c} u=(\alpha u)^{\prime}(t), \\
& M_{c}: D\left(M_{c}\right) \subset L_{\alpha}^{2}(H,(0,1)) \rightarrow L_{\alpha}^{2}(H,(0,1)), \\
& D\left(M_{c}\right)=\{u \in D(L):(\alpha u)(0)=0\}
\end{aligned}
$$

of the minimal operator $M_{0}$. It is clear that $M_{c}$ is a boundedly solvable extension of minimal operator $M_{0}$ and

$$
\begin{aligned}
& M_{c}^{-1} f(t)=\frac{1}{\alpha(t)} \int_{0}^{t} f(s) d s, f \in L_{\alpha}^{2}(H,(0,1)), \\
& M_{c}^{-1}: L_{\alpha}^{2}(H,(0,1)) \rightarrow L_{\alpha}^{2}(H,(0,1)) .
\end{aligned}
$$

Indeed, for any $f \in L_{\alpha}^{2}(H,(0,1))$ we have

$$
\begin{aligned}
\left\|\frac{1}{\alpha(t)} \int_{0}^{t} f(s) d s\right\|_{L_{\alpha}^{2}(H,(0,1))}^{2} & =\int_{0}^{1} \alpha(t) \frac{1}{\alpha^{2}(t)}\left\|\int_{0}^{t} f(s) d s\right\|_{H}^{2} d t \\
& \leq \int_{0}^{1} \frac{1}{\alpha(t)}\left(\int_{0}^{t} \frac{1}{\sqrt{\alpha(s)}} \sqrt{\alpha(s)}\|f(s)\|_{H} d s\right)^{2} d t \\
& \leq \int_{0}^{1} \frac{d t}{\alpha(t)}\left(\int_{0}^{1} \frac{d s}{\alpha(s)}\right)\left(\int_{0}^{1}\|f(s)\|_{H}^{2} \alpha(s) d s\right) \\
& =\left(\int_{0}^{1} \frac{d t}{\alpha(t)}\right)^{2}\|f\|_{L_{\alpha}^{2}(H,(0,1))}^{2}
\end{aligned}
$$

Now assumed that $\widetilde{M}$ is a solvable extension of the minimal operator $M_{0}$ in $L_{\alpha}^{2}(H,(0,1))$. In this case it is known that the domain of $\widetilde{M}$ can be written as a direct sum

$$
D(\widetilde{M})=D\left(M_{0}\right) \oplus\left(M_{c}^{-1}+K\right) V,
$$

where $V=\operatorname{Ker} M_{0}^{*}, K \in L(H)$ (see [4], [5]).

It is easily to see that

$$
\operatorname{KerM}_{0}^{*}=\left\{\frac{1}{\alpha(t)} f: f \in H\right\} .
$$

Therefore each function $u \in D(\widetilde{M})$ can be written in following form

$$
u(t)=u_{0}(t)+M_{c}^{-1}\left(\frac{1}{\alpha(t)} f\right)+\frac{1}{\alpha(t)} K f, u_{0} \in D\left(M_{0}\right), f \in H .
$$

And from this we have

$$
(\alpha u)(t)=\left(\alpha u_{0}\right)(t)+\int_{0}^{t} \frac{d s}{\alpha(s)} f+K f, f \in H .
$$


Hence, following equalities

$$
\begin{aligned}
& (\alpha u)(0)=K f \\
& (\alpha u)(1)=\left(\int_{0}^{1} \frac{d s}{\alpha(s)}+K\right) f .
\end{aligned}
$$

From these relations it is obtained that

$$
\left(\int_{0}^{1} \frac{d s}{\alpha(s)}+K\right)(\alpha u)(0)=K(\alpha u)(1) .
$$

Then the last equality can be written in form

$$
(B+E)(\alpha u)(0)=B(\alpha u)(1),
$$

where

$$
B=\left(\int_{0}^{1} \frac{d s}{\alpha(s)}\right)^{-1} K
$$

On the other hand note that the uniquenses of the operator $B \in L(H)$ is clear from [4], [5]. Therefore, $\widetilde{M}=M_{B}$. This completes of necessary part of assertion.

On the contrary, if $M_{B}$ is a operator generated by $m(\cdot)$ and boundary condition

$$
(B+E)(\alpha u)(0)=B(\alpha u)(1),
$$

then $M_{B}$ is boundedly invertible and

$$
\begin{aligned}
& M_{B}^{-1}: L_{\alpha}^{2}(H,(0,1)) \rightarrow L_{\alpha}^{2}(H,(0,1)), \\
& M_{B}^{-1} f(t)=\frac{1}{\alpha(t)} \int_{0}^{t} f(s) d s+B \int_{0}^{1} f(s) d s, f \in L_{\alpha}^{2}(H,(0,1)) .
\end{aligned}
$$

Consequently, assertion of theorem for the boundedly solvable extension of the minimal operator $M_{0}$ is true.

The extension $\widetilde{L}$ of the minimal operator $L_{0}$ is boundedly solvable in $L_{\alpha}^{2}(H,(0,1))$ if and only if the operator $\widetilde{M}=U^{-1} \widetilde{L} U$ is a boundedly solvable extension of the minimal operator $M_{0}$ in $L_{\alpha}^{2}(H,(0,1))$. Then $u \in D(\widetilde{L})$ if and only if $U^{-1} u \in D(\widetilde{M})$.

Since $\widetilde{M}=M_{B}$ for some $B \in L(H)$, then we have

$$
(B+E)\left(\alpha U^{-1} u\right)(0)=B\left(\alpha U^{-1} u\right)(1) .
$$

This completes the proof of theorem.

\section{Spectrum of boundedly solvable extensions}

In this section the structure of spectrum of boundedly solvable extensions of the minimal operator $L_{0}$ in $L_{\alpha}^{2}(H,(0,1))$ will be investigated. Firstly, prove the following result.

Theorem 3.1. If $L_{B}$ is a boundedly solvable extension of the minimal operator $L_{0}$ and $M_{B}=U^{-1} L_{B} U$ corresponding boundedly solvable extension of the minimal operator $M_{0}$, then it is true $\sigma\left(L_{B}\right)=\sigma\left(M_{B}\right)$.

Proof. Consider the following problem to spectrum for any boundedly solvable extension $L_{B}$ in $L_{\alpha}^{2}(H,(0,1))$, that is

$$
L_{B} u=\lambda u+f, \lambda \in \mathbb{C}, f \in L_{\alpha}^{2}(H,(0,1)) .
$$

From this it is obtained that

$$
\left(L_{B}-\lambda E\right) u=f \text { or }\left(U M_{B} U^{-1}-\lambda E\right) u=f .
$$

Then we have

$$
U\left(M_{B}-\lambda\right) U^{-1}(u)=f .
$$

Therefore, the validity of the theorem is clear.

Now prove the main theorem on the structure of spectrum. 
Theorem 3.2. The spectrum of the boundedly solvable extension $L_{B}$ of the minimal operator $L_{0}$ in $L_{\alpha}^{2}(H,(0,1))$ has the form

$$
\begin{aligned}
\sigma\left(L_{B}\right) & =\left\{\lambda \in \mathbb{C}: \lambda=\left(\int_{0}^{1} \frac{d s}{\alpha(s)}\right)^{-1}\left(\ln \left|\frac{\mu+1}{\mu}\right|+i \arg \left(\frac{\mu+1}{\mu}\right)+2 n \pi i\right),\right. \\
\mu & \in \sigma(B) \backslash\{0,-1\}, n \in \mathbb{Z}\} .
\end{aligned}
$$

Proof. By Theorem 3.1. for this it is sufficiently the investigate the spectrum of the corresponding boundedly solvable extension $M_{B}=$ $U^{-1} L_{B} U$ of the minimal operator $M_{0}$ in $L_{\alpha}^{2}(H,(0,1))$.

Now consider the following problem to spectrum for the extension $M_{B}$, that is,

$$
M_{B} u=\lambda u+f, \lambda \in \mathbb{C}, f \in L_{\alpha}^{2}(H,(0,1)) .
$$

Then

$$
(\alpha u)^{\prime}(t)=\lambda u(t)+f(t), t \in(0,1)
$$

with boundary condition

$$
(B+E)(\alpha u)(0)=B(\alpha u)(1) .
$$

It is clear that a general solution of the above differential equation has the form

$$
u(t ; \lambda)=\frac{1}{\alpha(t)} \exp \left\{\lambda \int_{0}^{t} \frac{d s}{\alpha(s)}\right\} f_{0}+\frac{1}{\alpha(t)} \int_{0}^{t} \exp \left\{\lambda \int_{s}^{t} \frac{d \tau}{\alpha(\tau)}\right\} f(s) d s, f_{0} \in H
$$

From this and boundary condition it is obtained that

$$
\left(E+B\left(1-\exp \left\{\lambda \int_{0}^{1} \frac{d s}{\alpha(s)}\right\}\right)\right) f_{0}=B\left(\int_{0}^{1} \exp \left\{\lambda \int_{s}^{1} \frac{d \tau}{\alpha(\tau)}\right\} f(s) d s\right)
$$

In case when $\lambda_{m} \int_{0}^{1} \frac{d s}{\alpha(s)}=2 m \pi i, m \in \mathbb{Z}$, from the last relation it is established that

$$
f_{0}^{(m)}=B\left(\int_{0}^{1} \exp \left\{\lambda_{m} \int_{s}^{1} \frac{d \tau}{\alpha(\tau)}\right\} f(s) d s\right), m \in \mathbb{Z} .
$$

Consequently, in this case the resolvent operator of $M_{B}$ is in form

$$
\begin{aligned}
R_{\lambda_{m}}\left(M_{B}\right) f(t) & =B\left(\frac{1}{\alpha(t)} \exp \left\{\lambda_{m} \int_{0}^{t} \frac{d s}{\alpha(s)}\right\} \int_{0}^{1} \exp \left\{\lambda_{m} \int_{s}^{1} \frac{d \tau}{\alpha(\tau)}\right\} f(s) d s\right) \\
& +\frac{1}{\alpha(t)} \int_{0}^{t} \exp \left\{\lambda_{m} \int_{s}^{t} \frac{d \tau}{\alpha(\tau)}\right\} f(s) d s, m \in \mathbb{Z} .
\end{aligned}
$$

Now assumed that $\lambda \neq 2 m \pi i, m \in \mathbb{Z}$. Then from the mentioned above equation for $f_{0} \in H$ we have

$$
\begin{aligned}
& \left(B-\left(1-\exp \left\{\lambda \int_{0}^{1} \frac{d s}{\alpha(s)}\right\}\right)^{-1} E\right) f_{0} \\
& =\left(1-\exp \left\{\lambda \int_{0}^{1} \frac{d s}{\alpha(s)}\right\}\right)^{-1} B\left(\int_{0}^{1} \exp \left\{\lambda \int_{0}^{1} \frac{d \tau}{\alpha(\tau)}\right\} f(s) d s\right), \\
& f_{0} \in H, f \in L_{\alpha}^{2}(H,(0,1)) .
\end{aligned}
$$

Then $\lambda \in \sigma\left(M_{B}\right)$ if and only if

$$
\mu=\left(1-\exp \left\{\lambda \int_{0}^{1} \frac{d s}{\alpha(s)}\right\}\right)^{-1} \in \sigma(B)
$$

In this case since $\mu \neq 0$,

$$
\exp \left\{\lambda \int_{0}^{1} \frac{d s}{\alpha(s)}\right\}=\frac{\mu+1}{\mu}, \mu \in \sigma(B), \mu \neq-1 .
$$


Then

$$
\lambda=\left(\int_{0}^{1} \frac{d s}{\alpha(s)}\right)^{-1}\left(\ln \left|\frac{\mu+1}{\mu}\right|+i \arg \left(\frac{\mu+1}{\mu}\right)+2 n \pi i\right), n \in \mathbb{Z} .
$$

Example 3.1. All boundedly solvable extensions $L_{k}$ of the minimal operator $L_{0}$ in $L_{\alpha}^{2}(0,1), \alpha(t)=t^{p}, p<1,0<t<1$, generated by differential expression

$$
l(u)=\left(t^{p} u(t)\right)^{\prime}+a(t) u(t), p<1,0<t<1, \frac{a(t)}{t^{p}} \in L^{1}(0,1)
$$

are generated by differential expression $l(\cdot)$ and boundary condition

$$
(k+1)\left(\alpha U^{-1} u\right)(0)=k\left(\alpha U^{-1} u\right)(1), k \in \mathbb{C},
$$

where $U(\cdot, \cdot)$ are the corresponding evolution operators. In this case the spectrum $\sigma\left(L_{k}\right)$ of the extension $L_{k}, k \neq 0,-1$ is in form

$$
\sigma\left(L_{k}\right)=\left\{\lambda \in \mathbb{C}: \lambda=(1-p) \ln \left|\frac{k+1}{k}\right|+i \arg \left(\frac{k+1}{k}\right)+2 n \pi i, n \in \mathbb{Z}\right\} .
$$

\section{Conclusion}

It is known that problem on the solvability of the degenerate differential equations with corresponding boundary conditions in finite and infinite regions is main subject in mathematical literature always (for detail informations see [1]).

It is noted that the general form of boundedly solvable extensions of some densely defined closed operator in Hilbert space has been found by M. I. Vishik. In our work using the techniques of mentioned above theory a parametrization of boundedly solvable extensions of the minimal operator generated by degenerate differential-operator expression for first order in the weighted Hilbert space of vector-functions at finite interval is investigated. Lastly, the structure of spectrum of these type extensions is given.

Point out that the general form and spectral analysis of subclasses of differential operators in Banach spaces are main research topics in operator theory.

\section{References}

[1] A. Favini, A. Yagi, Degenerate Differential Equations in Banach Spaces, Marcel Dekker, Inc., New-York-Basel-Hong Kong, 1999.

[2] J. A. Goldstein, Semigroups of Linear Operators and Applications, Oxford University Press, New York and Oxford, 1985, 245 pp.

[3] L. Hörmander, On the theory of general partial differential operators, Acta. Math. 94 (1955), 162-166.

[4] M. I. Vishik, On linear boundary problems for differential equations, Doklady Akad. Nauk SSSR (N.S) 65 (1949), 785-788.

[5] M. I. Vishik, On general boundary problems for elliptic differential equations, Amer. Math. Soc. Transl. II 24 (1963), 107-172. 